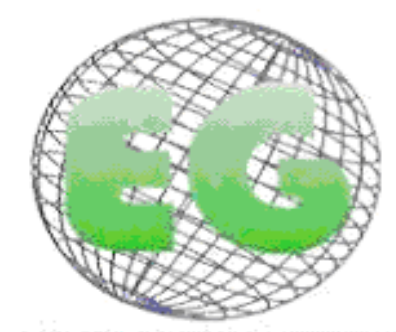

ISSN 1695-6141

N24
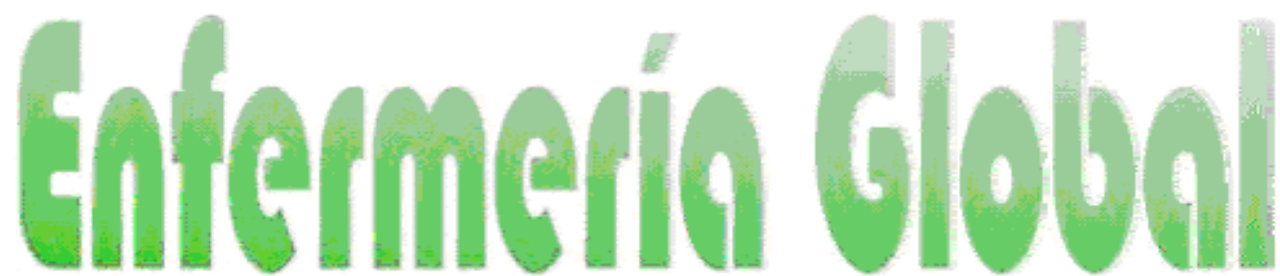

Revista electrónica trimestral de Enfermería

Octubre 2011

www.um.es/egloball

\title{
La calidad de vida profesional de las enfermeras en geriatría
}

Evaluation of the professional quality of life of geriatric nurses

\author{
*Hanzeliková Pogrányivá, A. **García López, MV., ***Pomares Martínez, M., \\ ${ }^{* * * *}$ Pardo Fernández, MJ., *****Del Monte Paz, J.
}

\begin{abstract}
*DUE. Magíster en Investigación en Cuidados. Hospital Virgen del Valle. ${ }^{* *}$ DUE. Lcda. en Antropología. Profesora Titular Escuela Universitaria de Enfermería de Castilla la Mancha. ${ }^{* *}$ DUE. Lcda. en Antropología. Hospital Virgen del Valle. ${ }^{* * * *}$ DUE. Subdirectora de Enfermería del Complejo Hospitalario. ${ }^{* * * * D U E . ~ H o s p i t a l ~}$ Virgen del Valle. Toledo.
\end{abstract}

(El presente trabajo fue presentado en el XVIII Congreso Nacional de la Sociedad Española de Enfermería Geriátrica y Gerontológica Murcia 07-08 abril 2011 como comunicación oral)

\begin{abstract}
Palabras clave: Calidad de vida profesional; Geriatría; Enfermería; Hospital. Keywords: Quality of Professional Life; nursing; geriatrics; hospital, care.
\end{abstract}

\section{RESUMEN}

Conocer la calidad de vida laboral de los trabajadores es muy importante porque tiene relación con el rendimiento laboral, la obtención de mejores resultados y una elevada productividad.

Objetivo: Conocer la Calidad de Vida Profesional percibida por las enfermeras en el Hospital Geriátrico Virgen del Valle de Toledo.

Metodología: Diseño: Estudio descriptivo transversal. Ámbito de estudio: Hospital geriátrico de Toledo. Población del estudio: Todas las enfermeras asistenciales del hospital un total de 69. Instrumento de medida: Se ha utilizado el cuestionario Calidad de Vida Profesional 35.

Resultados: En total se obtuvieron 45 respuestas $(65,2 \%)$. La valoración global medida sobre la Calidad de Vida Profesional percibida es baja. En relación con las tres dimensiones valoradas la media más alta se encuentra en la "motivación intrínseca" con 7,08, la "carga de trabajo" con una media de 6,56, siendo la dimensión con la media más baja el "apoyo directivo" con 5,59. En el análisis multivariante el apoyo directivo se mostró como el factor más influyente en la calidad de vida profesional con un $23 \%(P<0.001)$ seguido por la carga de trabajo con un $9 \%(P=0.01)$.

Conclusiones: Existe la percepción de baja calidad de vida profesional de las enfermeras. Con una adecuada 
motivación intrínseca, pero con cierta insatisfacción relacionada con la carga de trabajo y una escasa percepción de apoyo directivo. Los resultados obtenidos indican la necesidad de un cambio cultural organizativo basado en la participación, en la motivación y aumentando el apoyo directivo.

\section{ABSTRACT}

Knowing the quality of life of professionals is very important because it is related to job performance, better results, and greater productivity. Objetive To understand the Quality of Professional Life perceived by the nurses at the Geriatric Hospital Virgen del Valle of Toledo.

Methodology: Design: Descriptive cross-section study. Setting: Geriatric Hospital of Toledo. Study population: All healthcare nurses (69 in total) within the hospital. Measuring tool: the Quality of Professional Life 35 questionnaire was used as measuring instrument.

Results: In total, 45 responses were obtained (65.2\%). The overall mean score measured the perceived Quality of Professional Life to be at 5.29. In relation to the three dimensions evaluated in the study, the highest average found, in "intrinsic motivation" was 7.08, while "workload" had an average of 6.56, and "management support" was the dimension with the lowest average at 5.59. In the multivariate analysis, "management support" was shown to be the most influential factor in the Quality of Professional Life with $23 \%$ influence $(P<0.001)$, followed by workload with $9 \%(P=0.01)$.

Conclusions: There is a low perception of Quality of Professional Life and an adequate perception of intrinsic motivation, but with certain dissatisfaction related to workload as well as a scant perception of management support. The results indicate the necessity of an organizing cultural change based on participation, motivation, and increased management support.

\section{INTRODUCCIÓN}

La teoría de que las personas son el principal activo de las organizaciones se cumple de manera especial en el caso de las organizaciones sanitarias. Se puede afirmar que la calidad de los servicios prestados en las organizaciones sanitarias se relaciona directamente con la satisfacción de los profesionales que las integran ${ }^{(1,2)}$

La mejora de la satisfacción laboral y las condiciones de vida de los trabajadores debería ser un objetivo de las organizaciones en general, y de las sanitarias en particular dado que es imprescindible para garantizar el mantenimiento de la inversión en el capital humano y, por consiguiente, la mejor calidad de los servicios prestados ${ }^{(3)}$. Diferentes estudios demuestran la relación entre la satisfacción laboral de los profesionales y el grado de calidad de los cuidados prestados ${ }^{(4,5)}$. También hay relación entre la insatisfacción y absentismo, accidentes laborales, agresividad, cambios en el puesto de trabajo así como su incidencia directa en el nivel de estrés laboral y su percepción de la calidad de vida profesional ${ }^{(4,6)}$

En relación a las condiciones de vida en el trabajo hay dos términos habitualmente utilizados, el estrés laboral y el síndrome de burnout.

El estrés laboral fue definido por McGrath en 1970 como el desequilibrio percibido entre la demanda y la capacidad de contestación del individuo, en condiciones en las que el fracaso ante esa demanda supone importantes consecuencias ${ }^{(7)}$. El síndrome de burnout hace referencia al agotamiento, la despersonalización y la baja realización profesional, que puede aparecer especialmente en profesionales que trabajan con personas. Fue descrito la primera vez en 1974 por Freudenberg, y Maslach ${ }^{(7,8)}$. Éste es un resultante específico del estrés laboral crónico que suele aparecer en los profesionales que mantienen una relación de ayuda constante y directa con otras personas, tras soportar una sobrecarga de trabajo duradera y, normalmente, tras poner unas expectativas y una dedicación considerables en su trabajo. Con el tiempo, estos profesionales sufren los síntomas de cansancio emocional, despersonalización y falta de realización personal. Entre las variables asociadas a esta situación se señalan los factores ambientales, las características de la persona, las 
características del afrontamiento, los resultados previos de las experiencias de estrés y las consecuencias de esta situación excepcional para el individuo ${ }^{(9)}$.

El estrés laboral, el síndrome de burnout y su impacto han sido descritos en numerosas ocasiones en enfermería ${ }^{(10-14)}$

Tanto el estrés laboral como el síndrome de burnout influyen directamente en la satisfacción del profesional y en su percepción de la calidad de vida en el trabajo. Actualmente cobra importancia la calidad de vida profesional como objetivo de estudio desde la psicología de las organizaciones. Se considera una dimensión valiosa en sí misma y un objetivo de la intervención organizacional ${ }^{(15)}$. La calidad de vida profesional se relaciona con la satisfacción profesional. La satisfacción profesional es el grado de bienestar que experimenta la persona - individuo con motivo de su trabajo. Por otra parte la calidad de vida profesional (CVP) se define como el sentimiento de bienestar que se deriva del equilibrio que el individuo percibe entre las demandas o cargas de la profesión y los recursos (psicológicos, organizacionales y relacionales) de que dispone para afrontar estas demandas. Así, el trabajador necesita conseguir un desarrollo óptimo de la esfera profesional, personal y familiar para poder hablar de CVP $^{(6)}$.

Actualmente, en el medio sanitario existen escasas actuaciones de política sanitaria que reconocen la importancia estratégica de mejorar la satisfacción de los profesionales de la salud.

El objetivo del presente estudio es conocer la calidad de vida profesional percibida del personal de enfermería del Hospital Geriátrico Virgen del Valle de Toledo (España). También se propone conocer la relación entre el "Apoyo Directivo", la "Motivación Intrínseca"” y la "Demanda de Trabajo" con la Calidad de Vida Global percibida. Además se pretende saber qué factores influyen en la variabilidad de la CVP global.

\section{MATERIAL Y MÉTODOS}

Diseño: Estudio descriptivo transversal, prospectivo.

Ámbito de estudio: Hospital geriátrico de Toledo (España).

Población del estudio: Todas las enfermeras del hospital, un total de 69. Se incluyeron todas las enfermeras que a fecha de 1 de enero de 2010 tenían nombramiento como propietarios, interinos $u$ otras situaciones. Se excluyeron del estudio las supervisoras.

Variables del estudio: Variable dependiente: "calidad de vida global percibida".

Variables independientes: tres dimensiones: apoyo directivo, motivación intrínseca, demanda de trabajo, las variables sociodemográficas (sexo, edad), situación laboral (interino, propietario, otras), el tiempo trabajado como enfermera, el tiempo trabajado como enfermera en geriatría y lugar de trabajo.

Instrumento de medida El instrumento de medida fue el cuestionario Calidad de Vida Profesional 35 (CVP 35). Este cuestionario está basado en el modelo de control de demanda de Karasek y esta validado en el idioma español ${ }^{(6,16,17)}$. Además del cuestionario, se recogieron características demográficas y laborales.

El cuestionario CVP-35 consta de 35 preguntas que se responden en una escala de 1 a 10, (entre nada y mucho donde «nada» (valores 1 y 2); «algo» (valores 3, 4 y 5); «bastante» 
(valores 6, 7 y 8) y «mucho» (valores 9 y 10). Las peguntas se agrupan en tres dimensiones: "apoyo directivo" (12 ítems), "cargas de trabajo" (12 ítems) y "motivación intrínseca"(10 ítems). Existe una pregunta que no se pueden englobar en estas categorías. Es la medida resumen de la percepción de CVP. Las subescalas de cada dimensión muestran una consistencia interna muy aceptable (alpha de Cronbach entre 0,75 y 0,86$)^{(6)}$.

Recogida de datos: Los datos se recogieron durante el mes de enero de 2010 (de 10 de enero hasta 30 ).

El cuestionario anónimo, junto con una carta de presentación del estudio, se entregó en los lugares del trabajo en un sobre cerrado. La encuesta se entregó a las enfermeras personalmente por la persona responsable de la recogida de datos.

Análisis estadístico: Los datos obtenidos se analizaron mediante: estadísticos descriptivos, análisis de la variancia ANOVA unifactorial, pruebas de $\mathrm{T}$ de Student y Análisis de Regresión simple y múltiple.

Consideraciones éticas: El estudio fue aprobado por la comisión de investigación y por la comisión de ética en el Complejo Hospitalario de Toledo. Antes de comenzar el estudio se obtuvo el permiso de la dirección de enfermería. Los participantes del estudio fueron informados sobre el método y el fin de la investigación. Se respetó la libre participación (voluntaria) de las enfermeras en el estudio. Una vez obtenida la información se documentó de manera anónima y se empleará exclusivamente para los fines de la investigación.

\section{RESULTADOS}

En total se obtuvieron 45 respuestas (65,2\%). En la Tabla 1 se exponen las características de sexo, estado civil y situación laboral de los que contestaron. El 88,9\% eran mujeres y el $11,1 \%$ hombres. El 46,7 \% de los sujetos eran propietarios de la plaza (fijos). El 33,3 \% era de contrato de larga duración (interino o comisión de servicios) y el resto (20\%) disponía de contratos de sustitución. El mayor porcentaje de la muestra (29\%) se encontraba en el rango de edad de 25-30 años. De las personas que contestaron el mayor porcentaje llevaba trabajando como enfermera en geriatría entre 1 y 6 años. El 46,7\% de la muestra disponía entre 0-9 años de experiencia como enfermeras. El $82,2 \%$ de los sujetos trabajaban en unidades de hospitalización, el 6,7\% en consultas y el 11,1\% en otros servicios (farmacia, laboratorio, unidad de radiología).

\section{Tabla 1: Datos sociodemográficos y laborales de los participantes}


Tabla 1: Datos sociodemográficos y laborales de los participantes

\begin{tabular}{|c|c|c|}
\hline & $\mathbf{N}$ & $\%$ \\
\hline \multicolumn{3}{|l|}{ Sexo } \\
\hline varón & 5 & 11,1 \\
\hline mujer & 40 & 88,9 \\
\hline Total & 45 & 100 \\
\hline \multicolumn{3}{|l|}{ Edad (años) } \\
\hline$<25$ & 2 & 4,4 \\
\hline $25-30$ & 13 & 28,9 \\
\hline $31-35$ & 8 & 17,8 \\
\hline $36-40$ & 4 & 8,9 \\
\hline $41-45$ & 6 & 13,3 \\
\hline $46-50$ & 9 & 20,0 \\
\hline $51-55$ & 3 & 6,7 \\
\hline Total & 45 & 100 \\
\hline \multicolumn{3}{|l|}{ Situación laboral } \\
\hline propietaria & 21 & 46,7 \\
\hline comisión & 2 & 4,4 \\
\hline interina & 13 & 28,9 \\
\hline otro & 9 & 20,0 \\
\hline Total & 45 & 100 \\
\hline \multicolumn{3}{|l|}{ Lugar de trabajo } \\
\hline unidad de hospitalización & 37 & 82,2 \\
\hline consulta & 3 & 6,7 \\
\hline otros & 5 & 11,1 \\
\hline Total & 45 & 100 \\
\hline \multicolumn{3}{|c|}{ Experiencia como enfrmera (años) } \\
\hline $0-9$ & 21 & 46,7 \\
\hline $10-20$ & 14 & 31,1 \\
\hline $21-33$ & 10 & 22,2 \\
\hline Total & 45 & 100 \\
\hline \multicolumn{3}{|c|}{ Tiempo trabajado en geriatría (años) } \\
\hline$<0,5$ & 6 & 13,3 \\
\hline $0,5-1$ & 4 & 8,9 \\
\hline $2-5$ & 12 & 26,7 \\
\hline $6-10$ & 8 & 17,8 \\
\hline $11-15$ & 4 & 8,9 \\
\hline $16-20$ & 3 & 6,6 \\
\hline$>20$ & 8 & 17,8 \\
\hline Total & 45 & 100 \\
\hline
\end{tabular}

En las tablas 2 y $\mathbf{3}$ se muestran los resultados de las preguntas del cuestionario.

TABLA 2: Resultados de los tres factores del cuestionario: apoyo directivo, cargas de trabajo, motivación intrínseca y calidad de vida en el trabajo.

\begin{tabular}{|llll|}
\hline & Media & $\begin{array}{l}\text { Desviación } \\
\text { típica }\end{array}$ & Varianza \\
\hline Factor apoyo directivo & 5,1197 & 1,21632 & 1,479 \\
\hline Factor carga de trabajo & 6,5636 & 1,38477 & 1,918 \\
\hline Factor motivación intrínseca & 7,8617 & 0,81449 & 0,663 \\
\hline Calidad de vida en el trabajo & 5,2913 & 2,35120 & 5,528 \\
\hline
\end{tabular}




\section{TABLA 3: Descripción de la media, desviación típica y varianza}

\begin{tabular}{|c|c|c|c|}
\hline Pregunta del cuestionario & Media & $\begin{array}{l}\text { Desviación } \\
\text { Típica }\end{array}$ & Varianza \\
\hline \multicolumn{4}{|l|}{ 1. Factor: Apoyo directivo } \\
\hline Posibilidad de promoción & 3,58 & 2,331 & 5,431 \\
\hline Mi empresa trata de mejorar la calidad de vida de mi puesto & 3,49 & 2,107 & 4,437 \\
\hline Satisfacción con el sueldo & 5,69 & 1,869 & 3,492 \\
\hline Reconocimiento de mi esfuerzo & 3,82 & 2,037 & 4,149 \\
\hline Recibo información de los resultados de mi trabajo & 3,89 & 2,308 & 5,328 \\
\hline Es posible que mis propuestas sean escuchadas y aplicadas & 5,42 & 2,509 & 6,295 \\
\hline Posibilidad de ser creativo & 4,84 & 2,067 & 4,271 \\
\hline Apoyo de mis jefes & 5,22 & 2,679 & 7,177 \\
\hline Tengo autonomía o libertad de decisión & 5,38 & 2,434 & 5,922 \\
\hline Posibilidad de expresar lo que pienso y necesito & 5,89 & 2,534 & 6,419 \\
\hline Variedad en mi trabajo & 5,56 & 2,302 & 5,298 \\
\hline Apoyo de mis compañeros 11 & 7,67 & 2,226 & 4,955 \\
\hline \multicolumn{4}{|l|}{ 2. Factor: Motivación intrínseca } \\
\hline Apoyo de mi equipo 35 & 6,87 & 2,430 & 5,904 \\
\hline Satisfacción con el tipo de trabajo & 6,11 & 1,910 & 3,646 \\
\hline Motivación (ganas de esforzarme) & 6,78 & 2,152 & 4,631 \\
\hline Ganas de ser creativo & 6,96 & 2,246 & 5,043 \\
\hline Me siento orgulloso de mi trabajo & 8,29 & 2,018 & 4,074 \\
\hline Capacitación necesaria para hacer mi trabajo actual & 8,56 & 1,455 & 2,116 \\
\hline Mi trabajo es importante para la vida de otras personas & 9,18 & 0,936 & 0,877 \\
\hline Apoyo de mi familia & 8,49 & 1,926 & 3,710 \\
\hline Estoy capacitado para hacer mi trabajo actual & 9,00 & 0,977 & 0,955 \\
\hline \multicolumn{4}{|l|}{ 3. Factor: Cargas de trabajo } \\
\hline Cantidad de trabajo que tengo & 7,93 & 1,250 & 1,564 \\
\hline Prisas y agobios por falta de tiempo para hacer mi trabajo & 7,38 & 2,631 & 6,922 \\
\hline Carga de responsabilidad & 8,62 & 1,556 & 2,422 \\
\hline Estrés (esfuerzo emocional) & 7,36 & 2,395 & 5,734 \\
\hline Presión que recibo para realizar la cantidad de trabajo & 6,80 & 2,262 & 5,118 \\
\hline Presión recibida para mantener la calidad de mi trabajo & 6,80 & 2,180 & 4,755 \\
\hline Interrupciones molestas & 6,22 & 2,645 & 6,995 \\
\hline Mi trabajo tiene consecuencias negativas para mi salud & 6,51 & 2,727 & 7,437 \\
\hline Falta de tiempo para mi vida personal & 5,62 & 2,741 & 7,513 \\
\hline Incomodidad física en el trabajo & 5,29 & 2,474 & 6,119 \\
\hline Conflictos con otras personas de mi trabajo & 3,67 & 2,541 & 6,455 \\
\hline Desconecto al acabar la jornada laboral & 6,27 & 2,824 & 7,973 \\
\hline Calidad de vida en el trabajo & 5,29 & 2,351 & 5,528 \\
\hline
\end{tabular}


La valoración global media sobre la Calidad de Vida Profesional percibida es de 5,29. En relación con las tres dimensiones valoradas la media más alta se encuentra en la "motivación intrínseca" con 7,86, la "demanda de trabajo" con una media de 6,56 siendo la dimensión con la media más baja el "apoyo directivo" con 5,11 (Tabla 2).

Los resultados de los análisis de la varianza Anova y las pruebas d T de Student nos indican los siguientes hallazgos:

No hubo diferencias significativas en la relación de edad y percepción global de calidad vida profesional, ni con ninguno de sus tres componentes ("carga de trabajo", "apoyo directivo" "motivación intrínseca").

Atendiendo la situación laboral y tiempo trabajado en geriatría tampoco se encontraron diferencias significativas en valoración de la calidad de la vida profesional, ni con ninguno de sus tres componentes.

Al estudiar la percepción de calidad de vida profesional se encontraron diferencias significativas con el lugar de trabajo. Los encuestados que trabajan en la unidad de hospitalización valoran más bajo la calidad de vida laboral $(4,84<7,40, P=0.018)$ que aquellos que trabajan en "otros" (farmacia, laboratorio, rayos). Destaca también que no sólo lo valoraron más bajo que otros, si no que es una valoración baja.

En cuanto al tiempo trabajado como enfermera y la calidad de vida profesional, el apoyo directivo y la carga de trabajo no hubo relación. Sin embargo existen diferencias significativas con la motivación intrínseca. Las enfermeras que tienen experiencia entre 2133 son los que más alto valoran la motivación intrínseca y los que menos son las enfermeras entre $10-20$ años $(8,16>7,35, P=0,015$.).

Al estudiar las características del lugar del trabajo se encontraron diferencias significativas en cuanto a la motivación intrínseca. Las enfermeras que más bajo valoran la "motivación intrínseca" son las que trabajan en las unidades de hospitalización y las que más alto son las enfermeras de "otros servicios $(7,44<8,82 ; P=0.005)$. $(P=0,016)$. No hubo diferencias significativas en cuanto al apoyo directivo y carga de trabajo según lugar de trabajo.

Los ítems con puntuación más baja fueron: mi empresa trata de mejorar la calidad de mi puesto 3,49 ( DT 2,107), recibo información de los resultados de mi trabajo 3, 89 (DT 2,308), posibilidad de promoción 3,58 (DT 2,33), conflictos con otras personas 3,67( DT2,541), reconocimiento de mi esfuerzo 3,82. (DT 2, 037). Estos ítems pertenecen al factor "apoyo directivo" salvo el ítem "conflictos con otras personas es del factor "carga de trabajo.

Los ítems con puntuación más alta fueron: mi trabajo es importante para la vida de otras personas 9,18 (DT 0,936), estoy capacitado para hacer mi trabajo actual 9,00, (DT 0,977), capacitación necesaria para hacer mi trabajo 8,56 (Dt 1,455), apoyo de mi familia 8,49 (Dt 1,926), me siento orgulloso de mi trabajo 8,29 (DT 2,018), cantidad de trabajo que tengo 7,93 (DT1, 250), y el estrés 7,36 (DT 2,395). Estos ítems pertenecen al factor "motivación intrínseca" salvo el ítem "estrés" que pertenece al factor "carga de trabajo".

Se realizó un análisis de regresión lineal para conocer la influencia de cada dimensión sobre la calidad global. El porcentaje de la CVP global que es explicado por el "apoyo directivo" es de 38\%,( $\mathrm{P}<0.001)$, por la "carga de trabajo" es de $20 \%(\mathrm{P}<0.01)$ y por la "motivación intrínseca" es de $13 \%(P=0.02)$ 
Analizando la influencia conjunta de las dimensiones sobre la calidad de vida global mediante el análisis de regresión múltiple se obtuvo un $47 \%$ de variancia explicada $(\mathrm{P}=0.01)$. La dimensión con más peso es "Apoyo directivo" 23\% ( $P<0.001)$ seguido de "carga de trabajo" con un 9\% ( $\mathrm{P}=0.01)$. La dimensión "motivación intrínseca" no contribuye de forma significativa a la explicación de la calidad de vida global.

Los resultados del análisis de regresión múltiple de la calidad de vida global y los ítems del cuestionario mostraron que la influencia conjunta de los ítems: Mi trabajo tiene consecuencias negativas para mi salud $(33,44 \%(P<0.001))$, Mi empresa trata de mejorar la calidad de vida de mi puesto $(22,15 \%(\mathrm{P}<0.001))$. Lo que tengo que hacer queda claro $(31$, $7 \%(P<0.001))$ y Desconecto al acabar la jornada laboral $(15,5 \%(P=0.01))$, explica un $70 \%$ de la variancia total de la calidad de vida global. $(P<0.001)$. Ningún otro ítem aportó información significativa.

\section{DISCUSIÓN}

El porcentaje de respuesta obtenido $(65,2 \%)$ ha sido adecuado para los objetivos del presente estudio y están en la línea de otros estudios realizados en ambiente sanitario. ${ }^{(2,6,}$ $16,18-22)$. Pero aunque se haya obtenido una tasa respuesta aceptable, al tratarse de cuestionarios anónimos, se desconocen las causas de no respuesta, pero no podemos descartar que pudiera existir un sesgo de no respuesta. Además, si se da el caso de que las personas menos satisfechas con su calidad de vida profesional no hubieran contestado la encuesta, se debería considerar que los resultados obtenidos fueran mejores que los reales.

\section{Calidad de vida profesional}

La percepción acerca de su propia calidad de vida profesional global por parte de las enfermeras es baja, alcanzando una media de 5.29 que corresponde solo a "algo" de calidad de vida según el CVP-35, esta variable tiene una relación directa con la calidad de vida profesional y la satisfacción laboral de los profesionales. Este resultado es similar a lo encontrado en diferentes estudios ${ }^{(16,24)}$.

La calidad de vida global fue valorada significativamente diferente dependiendo del lugar de trabajo que ocupaban los encuestados. Los encuestados que trabajan en la unidad de hospitalización valoran más bajo la calidad de vida laboral $(\mathrm{P}=0.018)$ que aquellos que trabajan en "otros" (farmacia, laboratorio, rayos). Este hallazgo pude relacionarse con los sistemas de turno de trabajo que tienen en las unidades de hospitalizaciones y el horario fijo de turno de mañana en los otros servicios repercute en la calidad de vida laboral.

\section{Apoyo directivo}

Dentro del factor de apoyo directivo es donde se obtuvieron las puntuaciones más bajas. El ítem con la media más baja fue: "mi empresa trata de mejorar la calidad de mi puesto" 3,49 (DT 2,107), La percepción de que la empresa no se preocupa por mejorar la calidad de los empleados exigiría reenfocar el interés de los directivos hacia las necesidades de los trabajadores, además de las de los usuarios de nuestros servicios. Se deberían mejorar las habilidades directivas, así como la comunicación y feedback entre directivos y empleados.

La posibilidad de promoción 3,58 (DT 2,33) ocupa la segunda posición de los ítems peor valorados, Este efecto desmotivador de la existencia de un «techo profesional» ha sido claramente demostrado en profesionales de enfermería en Estados Unidos ${ }^{(22)}$. La baja valoración de la posibilidad de promoción coincide también con lo observado en otros 
estudios ${ }^{(22,23)}$ y es el reflejo de la percepción que los profesionales tienen de un sistema sanitario que favorece el estancamiento y en el que no se promueve la competencia profesional. Las enfermeras en cuanto a oportunidades de promoción y ascenso son muy limitadas y que no son muy transparentes. El individuo espera reconocimiento por el trabajo realizado y las promociones son una manera de gratificación que guardan relación con las aspiraciones personales y valores, como también la percepción de equidad y justicia, y por lo tanto tienen gran influencia en la satisfacción laboral ${ }^{(24)}$

Hacia la misma dirección apunta la gran diferencia existente entre las puntuaciones de "ganas de ser creativo" 6,96 (DT2, 24) y "posibilidad de ser creativo"4,84(DT2, 06).

Este resultado se puede asociar con la ausencia del reconocimiento del grado alcanzado en su desarrollo profesional en relación con su competencia, formación y actividad; hasta ahora ausente en las organizaciones y a que algunas de las actividades de dirección dentro de las instituciones son delegadas, por lo tanto la discriminación en el ascenso favorece la insatisfacción. Estudios demuestran también, que la falta de promociones son uno de los aspectos del trabajo que más insatisfacción produce entre las/os enfermeras/os ${ }^{(25,26)}$

Los ítems "reconocimiento de mi esfuerzo" 3,82. (DT 2, 037) y "recibo información de los resultados de mi trabajo 3, 89 (DT 2,308), también ocupan los lugares peores valorados. Eso puede ser interpretado como si la alta capacitación y la complejidad del trabajo que realizan las enfermeras no fuera debidamente reconocida. Todos los datos referentes a los aspectos laborales negativos, la falta de reconocimiento del trabajo personal han sido señalados con anterioridad por otros autores ${ }^{(2,6)}$ y se relacionan con insatisfacción, agotamiento y desgaste en el trabajo.

Las bajas puntuaciones obtenidas en el factor apoyo directivo presenta un grupo de profesionales que no perciben que la organización sanitaria se aproxime a sus necesidades profesionales de participación, creatividad y reconocimiento.

\section{Motivación intrínseca}

Por el contrario los ítems que más alta puntuación obtienen son los relacionados con la motivación intrínseca. Los ítems obtienen mayor puntuación en este factor son: mi trabajo es importante para la vida de otras personas 9,18 (DT 0,936), estoy capacitado para hacer mi trabajo actual 9,00, (DT 0,977), capacitación necesaria para hacer mi trabajo 8,56 (Dt 1,455), apoyo de mi familia 8,49 Dt 1,926), me siento orgulloso de mi trabajo 8,29 (DT 2,018), y el estrés 7,36 (DT 2,395). Estos resultados son similares con los encontrados en otros estudios. $(20,23,27)$

La elevada percepción de la motivación intrínseca se ha observado en más ocasiones, también con el uso de otros instrumentos de medida ${ }^{(18)}$. Es posible hallar un «efecto techo" en las preguntas de esta categoría. La autovaloración tan positiva de la capacitación para el trabajo actual puede ser interpretada como positiva siempre que no lleve unida la idea de que no se necesita mejorar en esa capacitación.

Las diferencias encontradas en la valoración de motivación intrínseca y experiencia laboral como enfermera no se asemejan a la opinión generalizada de que los más jóvenes son los más motivados en las organizaciones. Según este estudio los que menos valoran la motivación intrínseca son las enfermeras con experiencia entre 10 y 20 años $(P=0,015)$. 
Las diferencias encontradas en la valoración de la "motivación intrínseca" según el lugar de trabajo indican que las enfermeras que más bajo valoran la "motivación intrínseca" son las que trabajan en las unidades de hospitalización $(P=0.005)$. No se encuentran resultados similares en otros estudios. Este hallazgo se podría explicar como el trabajo en una planta de geriatría de enfermos agudos donde la edad avanzada, las multipatologías, el alto grado de dependencia y el alto porcentaje de mortalidad de los pacientes influye en el grado de motivación de las enfermeras.

\section{Cargas de trabajo}

El ítem Conflictos con otras personas de mi trabajo obtuvo la puntuación más baja con un 3 , 67 (DT 2,541) dentro del factor cargas del trabajo. Con esta baja puntuación sobre los conflictos no esta en la línea de otros estudios dónde en los que se encontró que la

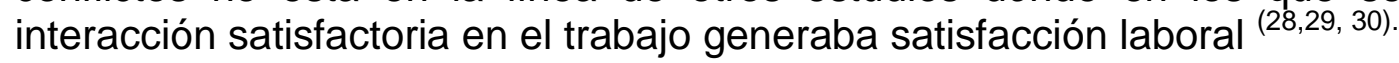

Las dimensiones que obtuvieron una alta puntación fueron: la cantidad de trabajo que tengo, carga de responsabilidad, estrés, prisas y agobios por falta de tiempo para hacer mi trabajo. Las condiciones de la calidad de vida laboral, la presencia de un buen clima laboral, un nivel de salud mental satisfactorio, disminuye la existencia de accidentes y enfermedades profesionales, además es necesario prestar atención al tema de la sobrecarga de trabajo diario, ya que es patente la presión asistencial excesiva y el riesgo de desarrollar el síndrome de Burnout. ${ }^{(27,31)}$

Está demostrado que la calidad de vida profesional se afecta negativamente cuando aumenta la presión asistencial incluso cuando ésta se compensa con incentivos económicos (32). Asimismo, la sobrecarga de trabajo ha sido identificada como una causa común de desmoralización e insatisfacción entre los profesionales 33,34.

El ítem "cantidad de trabajo que tengo" obtuvo la segunda puntuación más alta 7,93 (DT $1,25)$, lo que vendría a avalar la percepción subjetiva acerca de la escasez de plantilla. Sin embargo, cuando el conjunto de la dimensión "cargas de trabajo" no se encuentra por encima de 7, no estaría justificado un incremento de plantilla ${ }^{(23)}$. En el presente estudio, la puntuación del factor carga de trabajo es de 6,56, por lo que sería necesario analizar en profundidad cómo están siendo abordados los picos asistenciales a lo largo de la jornada laboral para confirmar este extremo.

Estudios han demostrado una relación negativa entre la carga de trabajo y la CVP ${ }^{(23)}$. Este estudio viene a avalar esta hipótesis al encontrar una correlación negativa entre las cargas de trabajo y la CVP.

Limitaciones del estudio: puede haber sesgos de información debidos al propio instrumento de medida, al valorar un concepto tan abstracto y subjetivo como la calidad de vida laboral.

Otra de las posibles limitaciones del estudio se puede corresponder a que el instrumento de medida solo recoge las percepciones, sin embargo no mide las expectativas. El conocimiento de las expectativas podría ser muy importante a la hora de introducir mejoras en el clima laboral.

El equipo investigador considera que el cuestionario CVP-35 es una herramienta muy útil y ya demostró su fiabilidad y validez en otros estudios ${ }^{(6)}$. Acerca al concepto de calidad de vida laboral que se había buscado para este estudio, sin embargo otros cuestionarios estaban más relacionados con los conceptos de burnout y el estrés laboral ${ }^{(8,9)}$ 


\section{CONCLUSIÓN}

Como conclusión de este estudio se destaca que los profesionales se encuentran en su lugar de trabajo con una alta responsabilidad, mucha carga de trabajo, prisas, agobios, y escaso apoyo directivo. Existe la percepción de baja calidad de vida profesional en las enfermeras. A pesar de eso los profesionales disponen de bastante motivación, apoyo familiar, orgullo por su trabajo y de una alta capacitación. Sería muy importante mejorar y optimizar los factores de trabajo que se identifiquen como influyentes en la calidad de vida profesional, y lograr así un clima laboral más agradable.

Para mejorar la percepción de la CVP hay que aumentar la percepción del apoyo directivo y disminuir la de cargas de trabajo. Este estudio podría aportar herramientas para conseguirlo. El conocimiento de las deficiencias detectadas sobre la CVP, proporciona a los directivos las áreas específicas donde se debe intervenir para conseguir cambios favorables para aumentar la percepción de la calidad de vida laboral. Eso podría redundar en una mayor eficacia de gestión de recursos porque con una adecuada cobertura de las necesidades de la enfermería se puede disminuir el estrés laboral, el síndrome de burnout y el absentismo laboral y al mismo tiempo mejorar los cuidados prestados.

Hay que destacar que la utilización de este instrumento para medir la percepción de calidad de la vida profesional debería ser una práctica tan habitual como el empleo de cuestionarios de satisfacción de los usuarios, ya que esto permitiría saber más sobre los trabajadores y su relación con su empresa y sus directivos para introducir mecanismos de corrección que eviten el agotamiento profesional.

El presente estudio se pretendió dotar con suficiente validez con el propósito de que sirva de aplicación para futuras investigaciones e intervenciones sobre el tema. Conocer la percepción que tienen las enfermeras sobre su calidad de vida laboral es un indicador. Aprovechando estos datos para instaurar programas de mejora que aumente la eficiencia y la cultura organizacional. Esto significa una planificación laboral motivadora y formativa, enriqueciendo el proceso de intercambio y comunicación entre directivos y enfermeras. Con eso se promovería una mayor participación en la toma de decisiones y aumentaría la confianza en el equipo directivo.

Este proyecto está en línea con las actuales investigaciones sobre la mejora de apoyo directivo en el ámbito sanitario para desarrollar medidas de mejora de un clima laboral satisfactorio. Los resultados pueden ser de interés científico de primera magnitud por su escasez de estudios similares en enfermería.

\section{AGRADECIMIENTOS}

Agradecemos a las enfermeras del Hospital Virgen del Valle su participación en el presente estudio.

\section{BIBLIOGRAFÍA}

1. Varo J. La calidad de la atención médica. Medicina Clinica 1995;104: 538-40.

2. Sibbald B, Enzer I, Cooper C, Rout U and Sutherland V. GP job satisfaction in 1987, 1990 and 1998: lessons for the future? Family Practice 2000; 17: 364-371. 
3. Donabedian A. Evaluating the quality of medical care.1966. The Mil bank Quarterly.2007;83:691-729.

4. Porter L, Steers R. Organizational work and personal factors in

Employee turnover and absenteeism. Psychological Bulletin. .1973:151-76.28.

5. Fernandez San Marin M,Villagrasa, GamoM, Vazquez J,Victoria M. Estudio de la satisfacción laboral y sus determinantes en los trabajadores sanitarios de una área de Madrid. Revista .Española de Salud Pública. 1995:487-97.

6. Cabezas P. C. La calidad de vida de los profesionales. Formación Médica Continada en Atención Primaria 2000;7(Supl 7):53-68.

7. Freudenberger H. Staff burnout. Journal of Social Issues $1974 ; 30: 159-65$

8. Maslach C, Schaufeli WB, Leiter MP. Job burnout. Annual Review Psychology 2001; 52: 397-422.

9. Cabezas P. C. Síndrome de desgaste profesional, estrés laboral y calidad de vida profesional. Formación Médica Continuada en Atención Primaria 1998;5:491-2.

10. Koivula M, Paunonen M, Laippala P.Burnout among nursing staff in two Finnish hospitals. Journal of Nursing Management 2000; 8: 149-58.

11. Clegg A .Occupational stress in nursing: a review of the literature. Journal of Nursing Management 2001; 9: 101-6.

12. Vahey DC, Aiken LH, Sloane DM, Clarke SP and Vargas D: Nurse Burnout and Patient Satisfaction Medical Care,2004 42(2), pp.II-57-II-66

13. Wu S, Zhu W, Wang Z, Wang M, Lan Y .Relationship between burnout and occupational stress among nurses in China. Journal of Advanced Nursing 2007; 59 (3) : 233-239.

14. Nayeri N.D, Negarandeh R, Vaismoradi M, Ahmadi F, Faghihzadeh S.

Burnout and productivity among Iranian nurses. Nursing Health Scienses. 2009 Sep;11(3):263-70

15 Meliá JL, Peiró JM. El cuestionario de satisfacción S10/12:estructura factorial, fiabilidad y validez. Revista de Psicología del Trabajo y de las Organizaciones 1989;4:179-87... .

16. García Sánchez S. La qualitat de vida profesional com a avantatge competitiu. Revista de Qualitat 1993; 11:4-9. 36.

17. Martín J, Cortés JA, Morente M, Caboblanco M, Garijo J, Rodríguez A. Características métricas del Cuestionario de Calidad de Vida Profesional (PQL -35) Gaceta Sanitaria. 2004;18:129-136. doi: 10.1157/13059283

18. Fernandez M, Moinelo A, Villanueva A, Andrade C, Rivera M, Gómez J. Satisfacción laboral de los profesionales de atención primaria del área 10 del Insalud de Madrid .Revista Española de Salud Publica.2000:139-47.

19. Alonso M, Iglesias A, Franco A. Percepción de la calidad de vida Profesional en un área sanitaria de Asturias. Atención Primaria.2002:483-9.

20. Cortés J, Martín J, Morente M, CaboblancoM ,Rodríguez JG. Clima laboral en atención primaria: ¿que hay que mejorar? .Atención Primaria.2003:288-95.

21. Goñi O., Ma A; Blanco P. R. Mํa ; Chamorro R. S.; Gómez G. I. Calidad de vida profesional de enfermeras y supervisores de enfermería del Hospital Ramón y Cajal y estrategias de desarrollo. Tesela 2008; 3. Available at http://0-www.indexcom.llull.uib.es/tesela/ts3/ts6610.php ( Accessed 10 Octuber 2010

22. Keith A, Coburn A, Mahoney E .Satisfaction with practice in a rural state: Perceptions of nurse practitioners and nurse midwives. Journal of the American Academy of Nurse Practitioners.1998:9-17.

23. Tzeng $\mathrm{H}$. The influence of nurses 'working motivation and job Satisfaction on intention to quit an empirical investigation in Taiwan International Journal of Nursing Studies.2002:86778.

24. Parra, S.; T. Paravic 2002. Satisfacción laboral en enfermeras/os que trabajan en el sistema de atención médica de urgencia (SAMU). Revista Ciencia y Enfermería. 8 (2) 
25. Courtney M, Yacopetti J, Walsh A. Queensland public sector nurse executives: job satisfaction and career opportunities. Australian Health Review 2001; 24 (2): 83-95.

26. Finn CP. Autonomy: an important component fo nurses' job satisfaction. International Journal of Nursing Studies 2001; 38 (3): 349-57.

27. Sanchez -González R, Alvarez-Nido R, Lorenzo-Borda S.; Calidad de vida profesional de los trabajadores de Atención Primaria del Área 10 de Madrid Revista de Medicina familiar y comunitaria.2003;13:291-6.

28.Nakayama Y, Aohda T, Katahira YA. Descriptive study on relationship between nurses' perception of their work and job satisfaction/retention. Seiroka Kango Daigaku Kiyo 1997; 23: $1-14$.

29. Adams B. Hospital nurses' job satisfaction, individual and organizational characteristics. Journal of Advanced Nursing 2000; 32 (3): 563-543.

30. Chaboyer W, Williams G, Corkill W, Creamer J. Predictors of job satisfaction in remote hospital nursing. Canadien Journal of Nursing Leadership 2000; 12 (2): 30-40.

31. Pla, O.; Hernández A. Artículos científicos: La enfermería y el estrés laboral. Avaliable at : www.enfervalencia.org/ei/articles/articulos04.htm. Accessed 17 september 2010

32. Grumbach K, Osmond D, Vranizan K, Jaffe D, Bindman AB.Primary care physicians' experience of financial incentives in managed-care systems. The New England Journal of Medicine 1998;339:1516-21

33. Smith R.Why are the doctors so unhappy? British Medical Journal 2001;322: 1073-4.

34. Huby G, Gerry M, McKinstry B, Porter M, Shaw J, Wrate R.Morale among general practitioners: qualitative study exploring relations between partnership arrangements, personal style and workload. British Medical Journal 2002; 325: 140-4. 